\title{
The Effect of Sonication on Acoustic Properties of Biogenic Ferroparticle Suspension
}

\author{
Arkadiusz JÓZEFCZAK ${ }^{(1)}$, Tomasz HORNOWSKI ${ }^{(1)}$, Anita KRÓL ${ }^{(1)}$, Matúš MOLČAN ${ }^{(2)}$, \\ Błażej LESZCZYŃSKI ${ }^{(1),(3)}$, Milan TIMKO ${ }^{(2)}$
}

(1) Institute of Acoustics, Faculty of Physics, Adam Mickiewicz University Umultowska 85, 61-614 Poznań, Poland; e-mail: aras@amu.edu.pl

(2) Institute of Experimental Physics, Slovak Academy of Sciences

Watsonova 47, 04001 Košice, Slovakia

(3) NanoBioMedical Centre, Adam Mickiewicz University

Umultowska 85, 61-614 Poznań, Poland

(received November 5, 2015; accepted December 17, 2015)

\begin{abstract}
Superparamagnetic iron oxide nanoparticles (SPION) synthesised chemically usually need the modification of the particle surface. Other natural sources of magnetic particles are various magnetotactic bacteria. Magnetosomes isolated from magnetotactic bacteria are organelles consisting of magnetite $\left(\mathrm{Fe}_{3} \mathrm{O}_{4}\right)$ or greigite $\left(\mathrm{Fe}_{3} \mathrm{~S}_{4}\right)$ crystals enclosed by a biological membrane. Magnetotactic bacteria produce their magnetic particles in chains. The process of isolation of magnetosome chains from the body of bacteria consists of a series of cycles of centrifugation and magnetic decantation. Using a high-energy ultrasound it is possible to break the magnetosome chains into individual nanoparticles - magnetosomes. This study presents the effect of sonication of magnetosome suspension on their acoustic properties, that is speed and attenuation of the sound. Acoustic propagation parameters are measured using ultrasonic spectroscopy based on FFT spectral analysis of the received pulses. The speed and attenuation of ultrasonic waves in magnetosome suspensions are analysed as a function of frequency, temperature, magnetic field intensity, and the angle between the direction of the wave and the direction of the field.
\end{abstract}

Keywords: magnetosomes; sonication; ultrasonic properties.

\section{Introduction}

Currently there is a great interest in the preparation of functional magnetic nanoparticles with respect to their application in various fields of biomedical diagnostics and nanosciences. The nanoparticles are usually synthesized in the process involving the co-precipitation of metal salts in alkali aqueous base. However, synthetic nanoparticles that have been used almost exclusively for biomedical applications do not fully match the high requirements with respect to a uniform size and morphology, biocompatibility, and high magnetisation capabilities. By contrast, biogenic magnetoparticles such as bacterial magnetosome particles derived from various magnetotactic bacteria, for instance, Magnetospirillum magnetotacticum, reveal a number of advantages. Biogenically-produced magnetosomes present unique features that are dif- ficult to obtain through the chemical synthesis of abiotically-produced magnetic nanocrystals (ARAUjo et al., 2015). Magnetosome particles are organelles consisting of magnetite crystals enclosed by the phospholipid membrane that offer a high degree of biocompatibility (CEYHAn et al., 2006; SUn et al., 2010). In most magnetosomes, the mineral core of magnetosomes consists of magnetic iron oxide $\left(\mathrm{Fe}_{3} \mathrm{O}_{4}\right)$. The membraneenveloped nanosized crystals are arranged in one or more chain-like structures. Thanks to the linear arrangement of the magnetosomes, the cells have a sufficiently large magnetic moment to be able to passively align along geomagnetic fields (magnetotaxis). Magnetosome magnetite crystals have high magnetisation, consistent species-specific nanometer sizes and morphologies, and based on a number of recent studies, appear to be biological compatible. Nanometersized magnetosomes are of great interest in biotech- 
nology since they have a large surface area which can be used for anchoring relatively large amounts of specific molecules and can be easily manipulated using an external magnetic field (ARAUJO et al., 2015). Unfortunately, it is difficult to obtain magnetosomes in large quantities (Sun et al., 2010) and therefore their properties have not been sufficiently characterised so far and no commercial application has as yet been recognized. Recently, a significant progress has been achieved in synthesis of magnetic particles by various bacterial strains. This allowed for the targeted modification of magnetic properties and various postsynthesis modifications (PROzOROv et al., 2007). Our first studies of the properties of magnetosome nanoparticles have shown that they may be considered as a good material for the biomedical applications in hyperthermia (Timko et al., 2009). The extensive knowledge about the behaviour of the new kind of magnetic nanoparticles - especially in the external magnetic field - is always required prior to their practical applications.

Ultrasound, depending on its energy, can be used both to manipulate the medium and to study its properties. In this work we employed both features of ultrasound. First, through the process called sonication, a sound energy was applied to break magnetosome chains into separate particles and then ultrasonic wave of small energy was used to probe the systems (both standard and sonicated magnetosomes) non-invasively in order to study the change in microstructure and intermolecular interactions. Many basic phenomena arise as ultrasound interacts with a particulate mixture and which ultimately determine the ultrasonic wave attenuation and phase velocity in the mixture and the dependence of these parameters on frequency. The contribution of each one to the wavenumber depends in part on the contrast between the physical properties of the suspended particles and those of the continuous phase (CHALLIS et al., 2005).

Additionally, the influence of the sonication on the magnetic properties of the magnetosomes was investigated. The obtained results can give some information on the magnetic, elastic, and thermal properties of magnetosome nanoparticles, which should help in their future applications in the medical diagnostics and therapies.

\section{Preparation of magnetosomes}

Bacterial magnetosomes studied in this work were synthesised by a biomineralisation process of magnetotactic bacteria Magnetospirillum, strain AMB-1 (gram-negative $\alpha$-proteobacterium). The magnetotactic bacteria are successfully cultivated in the laboratory conditions. They were grown micro-aerobically at $25^{\circ} \mathrm{C}$ in a growing medium for a period of 10 14 days.

Magnetotactic bacteria produce their magnetite particles $\mathrm{Fe}_{3} \mathrm{O}_{4}$ aligned intracellularly in a chain. The detailed description of the cultivation process of the bacteria is given in (Dzarova et al., 2011). The process of isolation of magnetosome chains from the body of bacteria consists of a series of cycles of sonication, centrifugation, and magnetic decantation. These isolation and purification procedures leave the surrounding membrane intact. Isolated magnetosome particles form stable, well-dispersed suspensions (standard magnetosomes sample IM) in water solution of HEPES (4-(2-hydroxyethyl)-1piperazineethanesulfonicacid) (Тімко et al., 2008). Using a high-energy ultrasound it is possible to break the magnetosome chains into individual nanoparticles (MOLCAN et al., 2014).

The sample denoted as SM was sonicated with an ultrasonic oscillator (Branson model 450) at the power of $200 \mathrm{~W}$ for 3 hours. Transmission electron microscopy (TEM) images of the samples are presented in Fig. 1. The crystalline magnetosome cores (magnetosomes without organic shell) of magnetite have a uniform size, shape, and exhibit magnetic properties in all their volume (GOJZEWski et al., 2012). Their size is around $40-45 \mathrm{~nm}$ in the diameter. Such magnetic par-

a)

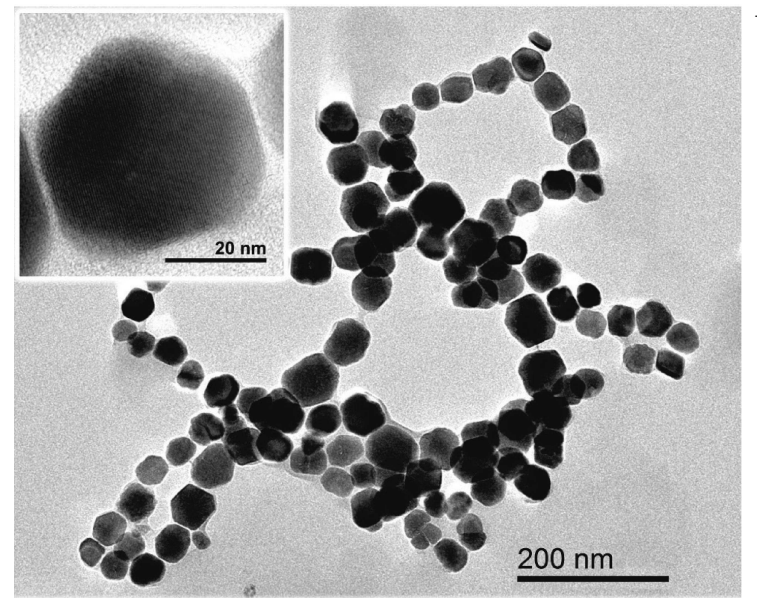

b)

c)

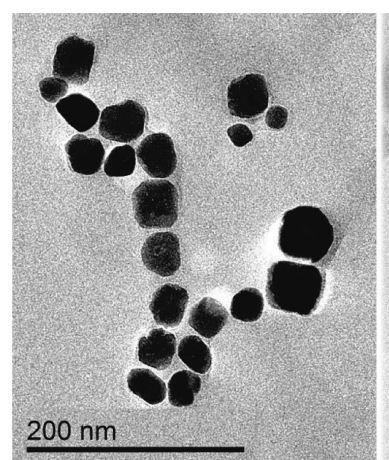

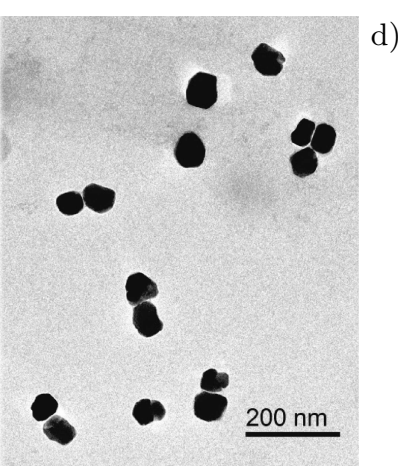

Fig. 1. Transmission electron micrographs of magnetosomes: a) $\mathrm{Fe}_{3} \mathrm{O}_{4}$ particle, b) isolated magnetosome chains, c) short magnetosome chains after sonication, d) individual nanoparticles after long sonication. 
ticles are small enough to form a single magnetic domain, which means that they still possess a permanent magnetic moment. A general problem with these systems is that chain-like assemblies of magnetic nanoparticles are not stable and often collapse into clusters and closed-ring structures (KIANI et al., 2015) to minimize their magnetic stray field energy (Timko et al., 2008). The single magnetosomes (monodomain nanomagnets) were obtained after sonication (Fig. 1d) and their suspension is more homogeneous.

\section{Magnetic properties of the studied magnetosomes}

Magnetisation measurements of the prepared magnetosome suspensions were carried out by Superconducting Quantum Interference Device (SQUID) Magnetometer of Quantum Design (JózEFCZAK et al., 2014). The dependence of magnetisation on the magnetic field (Fig. 2) measured at the room temperature show the ferromagnetic property of the samples. It was observed that synthetic magnetite nanocrystals had no remanence and no coercivity and showed superparamagnetic behaviour (HAN et al., 2007), however, as is seen in Fig. 2, the magnetosomes behave more like ferromagnets with small coercivity and remanence. In the nanometer scale magnetic properties are indeed size dependent. For ultrafine magnetite particles, the transition from superparamagnetic to ferromagnetic behavior occurs at a critical size $d$ of 25 $30 \mathrm{~nm}$ (HAN et al., 2007), while particles larger than $30 \mathrm{~nm}$ but smaller than $100 \mathrm{~nm}$ form stable single domains (BENNET et al., 2015). Thus, magnetosomes are ferromagnetic because their mean size is larger than $d$. In addition, the magnetic properties are affected by the morphology of the nanoparticles and their organisation (BENnET et al., 2015). Magnetosomes have a tendency to form chain-like structures and, after isolation, closeloop structures.

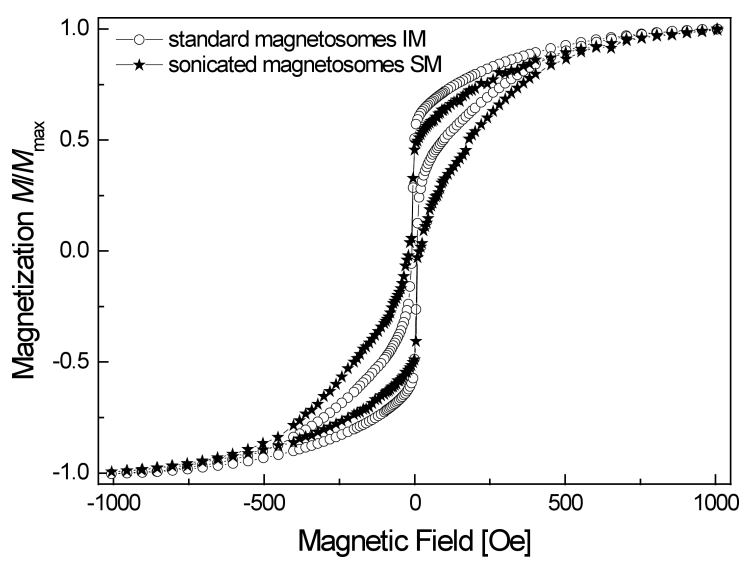

Fig. 2. Hysteresis loops for the studied samples: IM (standard) and SM (sonicated).
Figure 2 also clearly shows the effect of sonication on magnetic properties of magnetosome suspension. The hysteresis loop evidently looks different after sonication. This means that the magnetic hysteresis depends on the long-range (dipolar) interchain interactions and the shape anisotropy of the entire long chains, and not on the particle agglomeration or random dipolar interactions (Prozorov et al., 2007). The orderly arranged magnetosomes in the chains have strong interparticle dipolar interactions, so they exhibit a higher remanence than the separate magnetite nanoparticles. The magnetosomes organised in chains act as long dipoles with enhanced magnetic anisotropy along the chains (Ттмко et $l ., 2008$ ). It is the main reason why for the sonicated sample, containing partly individual particles, the remnant magnetisation is lower than that of the isolated sample (MOLCAN et al., 2014).

\section{Acoustic properties of the studied magnetosomes}

\subsection{Experimental methods}

The velocity and absorption of ultrasonic waves were measured by a continuous wave resonance method using a ResoScan ${ }^{\mathrm{TM}}$ System (Germany) apparatus in the absence of magnetic field and the pulse method when a sample was placed between pole pieces of the electromagnet. The ultrasonic velocity was determined from a series of resonance frequencies of the resonator cells. The fit of observed frequencies to a special transfer function of the ultrasonic resonator permits a determination of the required acoustic parameters. The measurement accuracy of the ultrasonic speed is about $\pm 0.01 \mathrm{~m} / \mathrm{s}$. The sound absorption given in relative magnitudes is corrected for energy losses observed in the cells when they are filled with water. The sound absorption coefficient is evaluated on the basis of the slope of the resonance curve at the resonance frequency. The relative error of the ultrasonic absorption coefficient is better than 5\%. The temperature of the samples is controlled to within $\pm 0.005 \mathrm{~K}$ by the Peltier thermostat.

For the study of the structural changes (aggregation process) under the influence of the magnetic field the ultrasonic pulse method was used. The broadband spectroscopy, as it is called, is based on application of short ultrasound pulses whose power spectra - determined by using a Fast Fourier Transform algorithm - are used to evaluate the sound velocity and attenuation. This method allows the measurement over a broadband frequency spectrum in a high dispersive media.

If the two acoustic signals are known, $p_{1}(t)$ and $p_{2}(t)$, for example, in two different positions of the wave, $z_{1}$ and $z_{2}$, the wave velocity and attenuation co- 
efficient can be calculated from the following formulas (Peters, Petit, 2003):

$$
\begin{aligned}
& v(f)=\frac{2 \pi f\left(z_{2}-z_{1}\right)}{\operatorname{Arg}\left(\frac{\widetilde{p}_{1}(f)}{\widetilde{p}_{2}(f)}\right)}=\frac{2 \pi f\left(z_{2}-z_{1}\right)}{\Delta \varphi}, \\
& \alpha(f)=\frac{\log \left(\left|\frac{\widetilde{p}_{1}(f)}{\widetilde{p}_{2}(f)}\right|\right)}{\left(z_{2}-z_{1}\right)},
\end{aligned}
$$

where $\widetilde{p}_{1}(f)$ and $\widetilde{p}_{2}(f)$ are the time Fourier transform of $p_{1}(t)$ and $p_{2}(t)$, respectively, and $\Delta \varphi$ is the absolute change of phase determined from the "unwrapped" phase spectrum of the signals. The latter was calculated using the method described in (Peters, Petit, 2003).

The ultrasonic measuring system consists of a measuring cell with ultrasonic transducer, ultrasonic pulser/receiver in the form of a standalone device, controlling computer, and thermostat. A block diagram of the experimental setup is shown in Fig. 3. Because of the unavailability of significant amounts of study material the measuring cell with a small volume $(1 \mathrm{ml})$ was constructed. The measuring cell is made of a nonmagnetic material, is small enough to fit into the restricted area between the pole pieces of the electromagnet and enable temperature control of the studied fluid. For generation of the ultrasonic pulses ultrasonic box with an integrated pulser and receiver was used. OPBOX 2.1 is the complete ultrasonic testing device, suitable for all ultrasonic measurements. The immersion broadband transducer (V324-SM, 0.25" element diameter, PANAMETRICS-OLYMPUS) was used. The suspension in the cell was in a direct contact with the piezoelectric plate. Ultrasonic pulses have a very low energy and they are unlikely to perturb the macroscopic state of the material through which the waves travel.

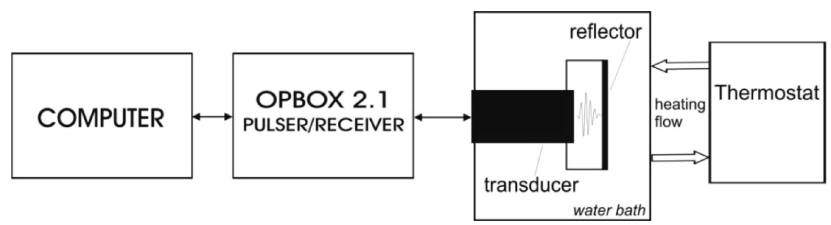

Fig. 3. Block diagram of the experimental setup.

In order to measure the effect of the magnetic field on the ultrasonic velocity and attenuation the measuring cell was placed between the poles of the electromagnet. The magnetic field was measured with the accuracy of $0.5 \%$ with a Resonance Technology RX21 type teslameter by placing the hall probe between the pole pieces of the electromagnet when the magnetic field was applied. The ultrasonic wave propagation parameters were measured during the ferrofluid exposure to the magnetic field. The accuracy of the velocity and attenuation measurement was in this case $0.1 \%$, and $5 \%$, respectively.

\subsection{Results of the ultrasonic measurements}

Figure 4 presents the effect of sonication on the ultrasound velocity in magnetosome suspensions, standard and sonicated. As it is seen in the figure, the speed of the sound is lower in the sonicated sample in comparison with the standard one. This can be explained by the increase in adiabatic compressibility - the stiffness of the medium containing the chains of particles is greater due to the magnetic dipole-dipole interaction between particles in the chain that favors straight chain orientation and the elastic contribution due to the bending stiffness of the actin-like cytoskeletal filament to which the magnetosomes are attached (KIANI et al., 2015). KIANI's et al. (2015) analysis shows that while both contributions are relevant, the bending stiffness of the filament can usually be expected to be the dominant part, with an about four-fold longer persistence length than due to the magnetic interaction alone.

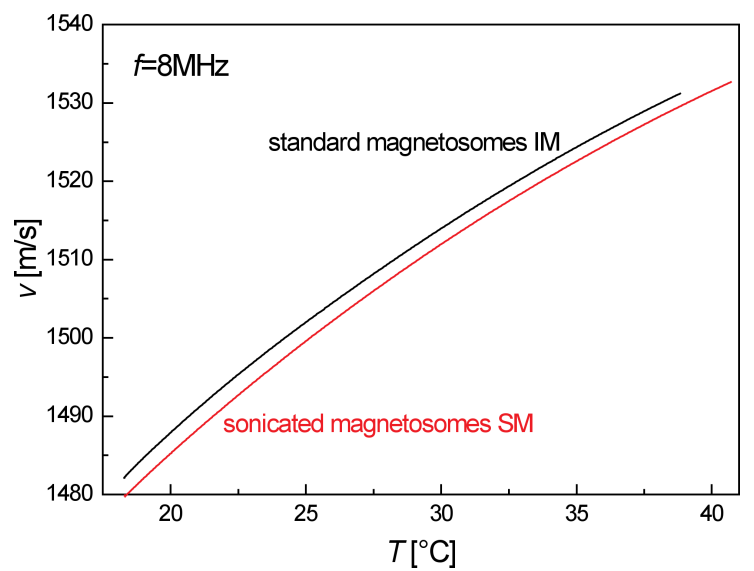

Fig. 4. Ultrasonic wave velocity $v$ as a function of temperature $T$ in standard and sonicated magnetosome suspensions measured by a resonance method for a wave of frequency $f=8 \mathrm{MHz}$.

The measurement of the ultrasound velocity and attenuation as a function of temperature and frequency of the wave in the magnetic field of different intensities was carried out using the pulse method and the FFT analysis of the signals. Figure 5 shows the dependence of the acoustical parameters on the frequency of the ultrasonic wave. It can be seen that there is a marked dispersion of the velocity and attenuation: the velocity increases from $1357 \mathrm{~m} / \mathrm{s}$ at $3 \mathrm{MHZ}$ to $1529 \mathrm{~m} / \mathrm{s}$ at $7 \mathrm{MHz}$, while relative attenuation (calculated with respect to the attenuation at $5 \mathrm{MHz}$ ) increases from 0.83 to 1.06 in the same frequency range. Such behavior is characteristic for the colloid suspension when the following condition is met: $k r \ll 1$ (DukHin, Goetz, 2002), where $k=2 \pi / \lambda$ is the wave number, and $r$ is the average nanoparticles' radius. For the ultrasonic wave of the megahertz frequency and particle's size on 


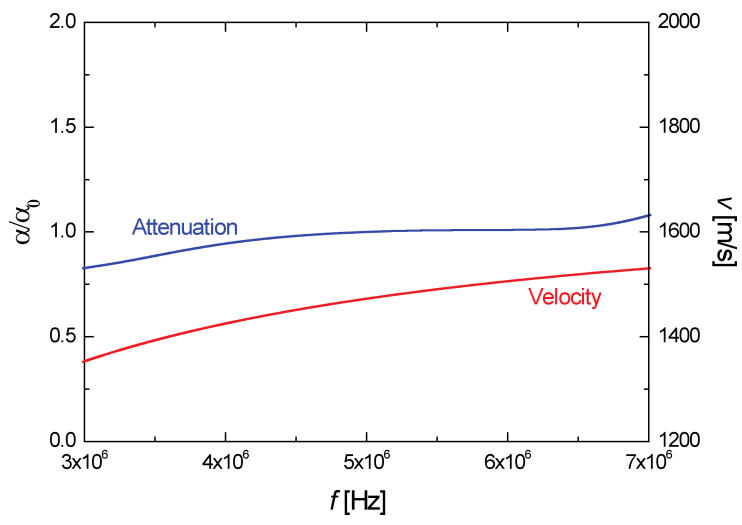

Fig. 5. Ultrasonic wave velocity $v$ as a function of frequency $f$ measured by the broadband ultrasonic spectroscopy in a standard magnetosomes sample for $T=283.6 \mathrm{~K}$.

the order of several nanometers the above condition is fulfilled.

The dependence of the ultrasonic velocity in standard as well as sonicated sample on the temperature for different intensities of the magnetic field is shown in Fig. 6. The decrease of the ultrasound velocity for sonicated magnetosome in comparison with the nonsonicated sample is not visible because the accuracy and resolution of the pulse method is significantly lower than that of the resonance technique. However, the dependence of the velocity results on the temperature is the same in the case of both methods of measurement.

There are two main mechanisms responsible for the dependence of ultrasonic velocity and attenuation on the magnetic field in magnetic fluids: magnetisation relaxation and internal chain dynamics. The former is responsible mainly for the behaviour of the ultrasound velocity in the magnetic field (OvCHINNIKOv, Sokolov, 2013), while the latter governs the dependence of the ultrasound attenuation on the magnetic field strength (SHLiomis et al., 2008).

Ovchinnikov and Sokolov's theory (Ovchinnikov, Sokolov, 2009; 2013) considers the propagation velocity of acoustic waves in a magnetic fluid under the influence of an external magnetic field. They assume magnetic fluid to be ideal with no attenuation whatsoever and the model of frozen-in magnetisation, i.e., the case where the magnetisation relaxation time is infinitely large. As a result the applied magnetic field $\mathbf{H}$ differs from the equilibrium magnetic field strength

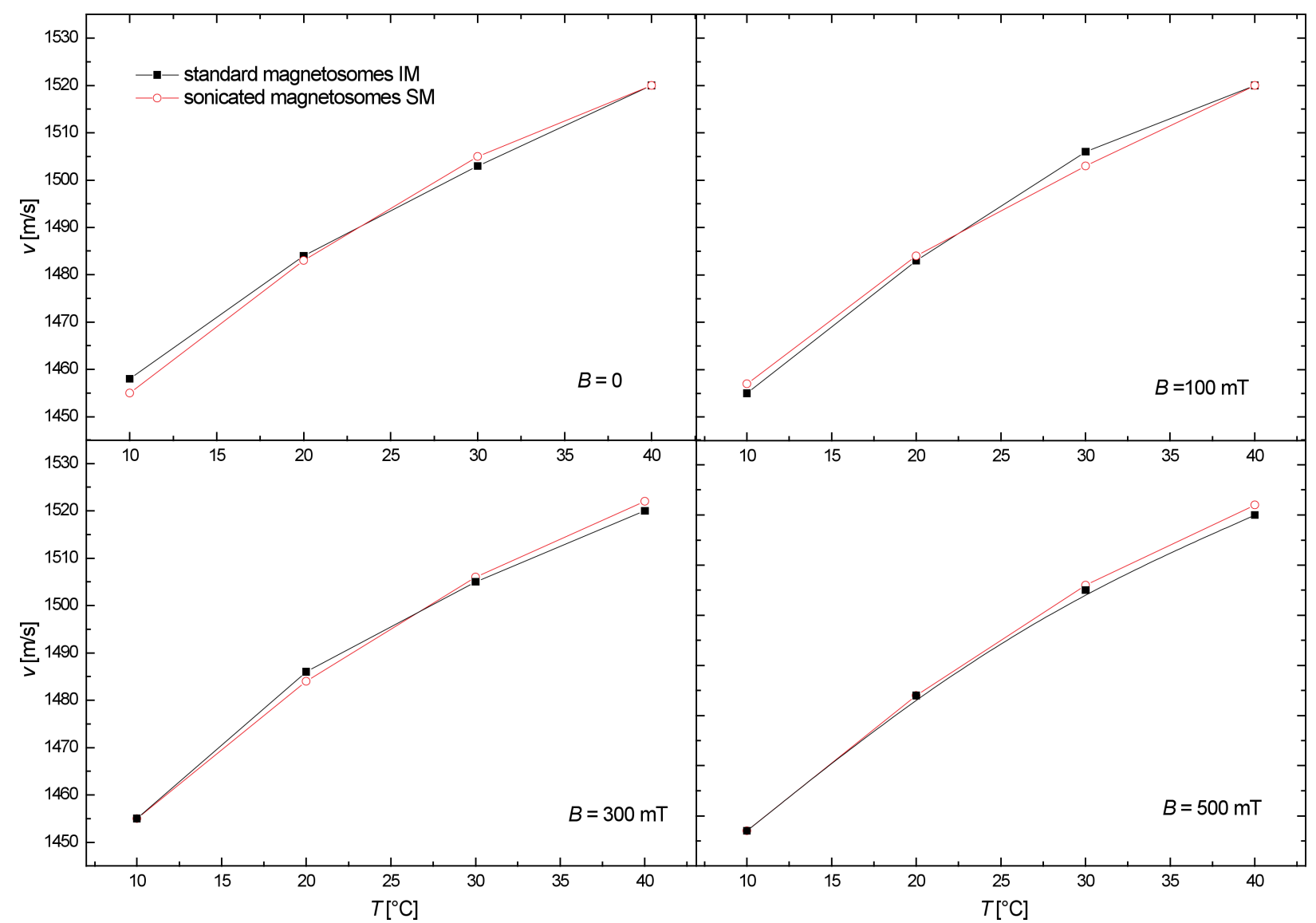

Fig. 6. Ultrasonic wave velocity $v$ as a function of temperature $T$ in standard and sonicated magnetosome suspension in both the absence and presence of the magnetic field for a wave of frequency $f=5 \mathrm{MHz}$. 
$\mathbf{H}^{\mathbf{e q}}$ in the fluid. Their assumption leads to the following expression for the ultrasound velocity in magnetic liquids:

$$
v=\left\{\frac{1}{2}\left(v_{0}^{2}+v_{A}^{2}\left(\frac{\beta_{\|}}{\beta_{\perp}}+1\right)+\sqrt{a^{*}}\right)\right\}^{1 / 2},
$$

where

$a^{*}=\left(v_{0}+v_{A}^{2}\left(\frac{\beta_{\|}}{\beta_{\perp}}-1\right)\right)^{2}+4 v_{0}^{2} v_{A}^{2}\left(1-\frac{\beta_{\|}}{\beta_{\perp}}\right) \sin ^{2} \theta$,

where $v_{0}$ is the ultrasonic velocity in the absence of a magnetic field, $v_{A}=m_{0} \sqrt{\beta_{\perp}} \cos \theta$ is the phase velocity of the Alfvén-type wave, $m_{0}=M_{0} / \rho$ is the specific magnetisation, $\beta_{\|}$and $\beta_{\perp}$ are the components of the diagonal tensor that determines the magnetoelastic properties of the magnetic fluid, and $\theta$ is the angle between the wave vector and the applied magnetic field. The specific magnetisation of the magnetic fluid as a function of the external magnetic field can be determined from the formula

$$
m_{0}=\frac{\phi M_{b}}{\rho} L(\xi)
$$

where $\rho$ is the density of the magnetic liquid, $\phi$, is the concentration of magnetite particles, $L(\xi)=$ $\operatorname{coth} \xi-1 / \xi$ is the Langevin function, $\xi=\mu_{0} m H / k_{B} T$ is the Langevin parameter, $H$ is the magnetic field strength, $k_{B}$ is the Boltzmann constant and $T$ is the absolute temperature. The magnetic moment $m=$ $M_{b} V$ of the particle is proportional to its volume, $V=\pi d^{3} / 6$ and the bulk magnetisation of the magnetic material $M_{b}$.

Figure 7 presents the prediction of ultrasound velocity as a function of the magnetic field strength (expressed as non-dimensional Langevin parameter, $\xi$ ) in magnetic fluid according to Eq. (3) for different concentrations of magnetite nanoparticles and the ultrasonic wave propagating parallel and perpendicular to the direction of the magnetic field. For the calculation the following typical results for the magnetic fluid were used: $\rho=1.2 \mathrm{~g} / \mathrm{cm}^{3}, d=10 \mathrm{~nm}, \beta_{\|}=2.6 \times 10^{5} \mathrm{~g} / \mathrm{cm}^{3}$, $\beta_{\perp}=7.2 \times 10^{5} \mathrm{~g} / \mathrm{cm}^{3}, M_{b}=480 \mathrm{G}$. As it is seen from the figure the effect of the magnetic field on the acoustical parameters of the magnetic fluid is visible only when the concentration of the magnetite grains is above $1 \%$.

The concentration of magnetite particles calculated from the magnetisation curve equals $0.035 \%$. It is too small for the magnetoacoustic effect to be observed given the accuracy and the resolution of both pulse and resonance methods of measurement. This conclusion is supported by Fig. 8 where the experimental values of the ultrasound velocity are shown together with the theoretical prediction based on Eq. (3). For the calculation of the theoretical curve the following values were used: $\rho=1.01 \mathrm{~g} / \mathrm{cm}^{3}, d=40 \mathrm{~nm}, \beta_{\|}=$ $2.6 \times 10^{5} \mathrm{~g} / \mathrm{cm}^{3}, \beta_{\perp}=7.2 \times 10^{5} \mathrm{~g} / \mathrm{cm}^{3}, M_{b}=480 \mathrm{G}$. a)

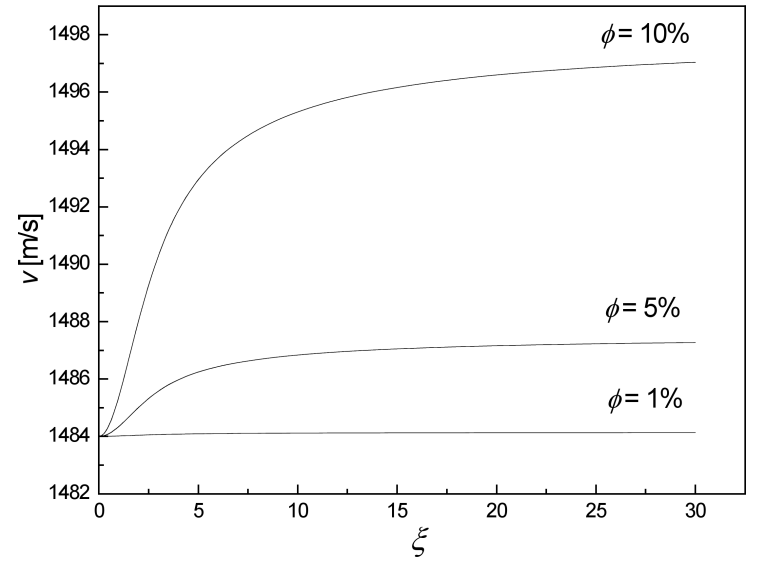

b)

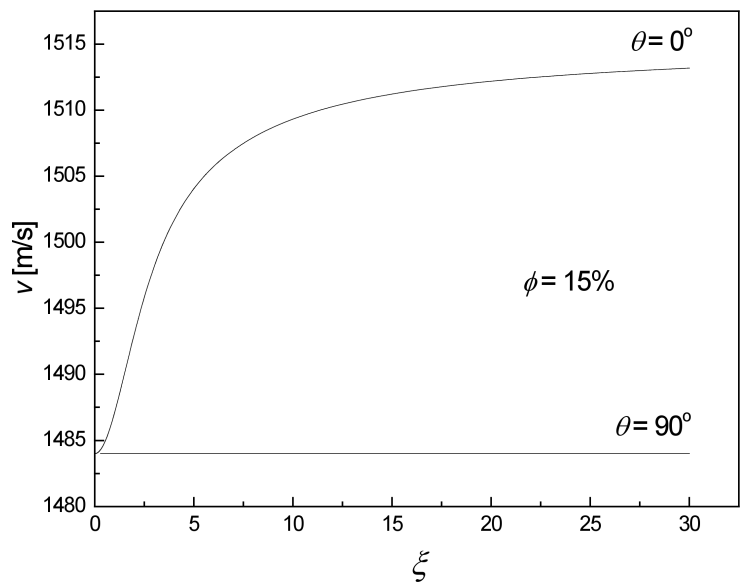

Fig. 7. Prediction of the ultrasound velocity as a function of the magnetic field strength (expressed as Langevin parameter $\xi$ ) in magnetic fluid according to the Eq. (3): a) for different concentration of magnetite particles and the wave propagating parallel to the direction of the magnetic field, and b) for the wave propagating parallel and perpendicular to the magnetic field in the magnetic fluid of $15 \%$ concentration of magnetite nanoparticles.

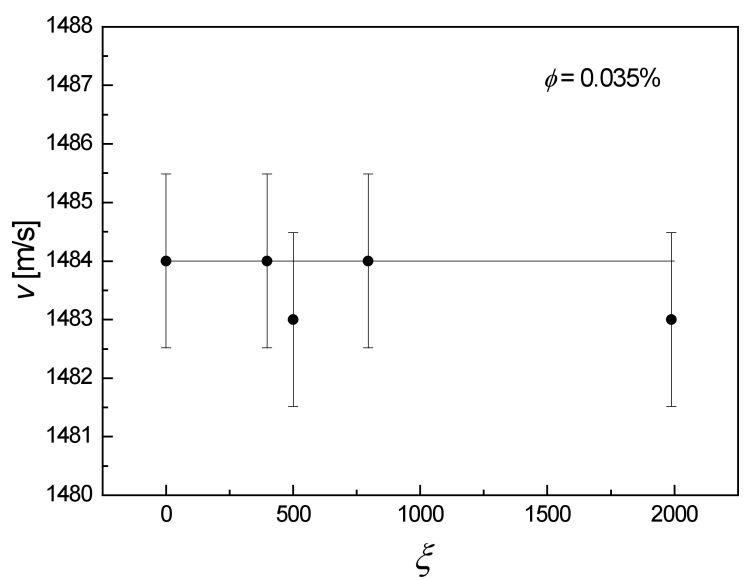

Fig. 8. Dependence of the ultrasound velocity on the magnetic field strength expressed as Langevin parameter $\xi$, in the sonicated magnetosome suspension.

The solid line was determined from Eq. (3). 


\section{Conclusion}

The study presents the effect of sonication of magnetosome suspensions on their magnetic and elastic properties. A high energy ultrasound wave was used to break magnetosome chains into separate particles. After sonication of the standard magnetosome suspension the single-magnetic-domain magnetosomes (monodomain nanomagnets) dispersed in a water solution of HEPES were obtained.

The arrangement of the single-magnetic-domain magnetosomes in chains cause each magnetosome moment to orient in parallel to the others along the chain axis, which leads to the maximum possible saturation and remanence magnetisation. After breaking the chains during the sonication the remnant and saturation magnetisation become lower than those of the isolated sample because of smaller interparticle dipolar interactions. As a result the hysteresis loop changes, which is observed in the experiment.

The acoustic study shows that the speed of the sound is lower in the sonicated sample in comparison with the standard one because the stiffness of the medium containing the chains of particles is greater due to the magnetic dipole-dipole interaction among particles in the chain. No change in the ultrasound velocity under the influence of the magnetic field was recorded. However, although the magnetoacoustic effect probably exists in magnetosome suspension, it may be too small in the studied samples (with a very low concentration of magnetosomes, $0.035 \%$ ) to be observed given the accuracy of the pulse method.

\section{Acknowledgment}

This work was supported by the Polish National Science Centre grant DEC-2011/03/B/ST7/00194 (A.J., T.H.), the European Social Fund (POKL.04. 03.00-00-015/12) (B.L.) and Slovak Academy of Sciences and Ministry of Education in the framework of projects VEGA No. 2/0043/12, 1/0861/12, Ministry of Education Agency for structural funds of EU in the frame of project Nos. 26110230097, M-era.Net project MACOSYS and COST RADIOMAG (M.M., M.T.).

\section{References}

1. Araujo A., Abreu F., Silva K., Bazylinski D., Lins U. (2015), Magnetotactic Bacteria as Potential Sources of Bioproduct, Marine Drugs, 13, 389-430, doi: $10.3390 / \operatorname{md} 13010389$.

2. Bennet M., Bertinetti L., Neely R.K., Schertel A., Kornig A., Flors C., Muller F.D., Schuler D., Klumpp S., Faivre D. (2015), Biologically controlled synthesis and assembly of magnetite nanoparticles, Faraday Discussions, 181, 71-83, doi: 10.1039/C4FD00240G.
3. Ceyhan B., Alhorn P., Lang C., Schüler D., Niemeyer C.M. (2006), Semisynthetic Biogenic Magnetosome Nanoparticles for the Detection of Proteins and Nucleic Acids, Small, 2, 1251-1255, doi: 10.1002/smll.200600282.

4. Challis R.E., Povey M.J.W., Mather M.L., Holmes A.K. (2005), Ultrasound techniques for characterizing colloidal dispersions, Rep. Prog. Phys., 68, 1541-1637, doi: 10.1088/0034-4885/68/7/R01.

5. Dukhin A.S., Goetz P.J. (2002), Ultrasound for characterizing colloids, Elsevier, New York.

6. Dzarova A., Royer F., Timko M., Jamon D., Kopcansky P., Kovac J., Choueikani F., GouzeWski H., Rousseau J.J. (2011), Magneto-optical study of magnetite nanoparticles prepared by chemical and biomineralization process, Journal of Magnetism and Magnetic Materials, 323, 1453-1459, doi: 10.1016/j.jmmm.2010.12.041.

7. Gojzewski H., Makowski M., Hashim A., KopcanSky P., Tomori Z., Timko M. (2012), Magnetosomes on surface: an imaging study approach, Scanning, 34, 159-169, doi: 10.1002/sca.20292.

8. Han L., Yang S.L.Y., ZhaO F., Huang J., Chang J. (2007), Comparison of magnetite nanocrystal formed by biomineralization and chemosynthesis, J. Magn. Magn. Matter., 313, 236-242, doi: 10.1016/j.jmmm.2007.01.004.

9. Józefczak A., Hornowski T., ZÁvišová V., Skumiel A., Kubovčíková M., Timko M. (2014), Acoustic wave in a suspension of magnetic nanoparticle with sodium oleate coating, J. Nanopart. Res., 16, 2271, doi: 10.1007/s11051-014-2271-z.

10. Kiani B., Faivre D., Klumpp S. (2015), Elastic properties of magnetosome chains, New Journal of Physics, 17, 043007, doi: 10.1088/1367-2630/17/4/043007.

11. Molcan M., Hashim A., Kovac J., Rajnak M., Kopcansky P., Makowski M., Gojzewski H., Molokac M., Hvizdak L., Timko M. (2014), Characterization of Magnetosomes After Exposure to the Effect of the Sonication and Ultracentrifugation, Acta Phys. Pol. A, 126, 198-199, doi: 10.12693/APhysPolA.126.198.

12. Ovchinnikov I.E., Sokolov V.V. (2009), Effect of an External Magnetic Field on the Propagation Velocities of Magnetoacoustic Waves in a Magnetic Fluid, Acoustical Physics, 55, 359-364, doi: 10.1134/S1063771009030117.

13. Ovchinnikov I.E., Sokolov V.V. (2013), Waves in Magnetic Fluids with Equilibrium and Frozen-In Magnetizations, Acoustical Physics, 59, 51-55, doi: 10.1134/S1063771012060115.

14. Peters F., Petit L. (2003), A broad band spectroscopy method for ultrasound wave velocity and attenuation measurement in dispersive media, Ultrasonics, 41, 357-363, doi: 10.1016/S0041-624X(03)00109-4.

15. Prozorov R., Prozorov T., Mallapragada S.K., Narasimhan B., Williams T.J., Bazylinski D.A. 
(2007), Magnetic irreversibility and the Verwey transition in nanocrystalline bacterial magnetite, Physical Review B, 76, 054406, doi: 10.1103/PhysRevB.76.054406.

16. Shliomis M., Mond M., Morozov K. (2008), Ultrasound Attenuation in Ferrofluids, Phys. Rev. Lett., 111, 074505, doi: 10.1103/PhysRevLett.101.074505.

17. Sun J., Tang T., Duan J., Xu P.-X., Wang Z., Zhang Y., Wu L., Li Y. (2010), Biocompatibility of bacterial magnetosomes: Acute toxicity, immunotoxicity and cytotoxicity, Nanotoxicology, 4, 271-283, doi: 10.3109/17435391003690531.
18. Timko M., Dzarova A., Kovac J., Skumiel A., Józefczak A., Hornowski T., GoJżewski H., Zavisova V., Koneracká M., Sprincova A., KopČAnskÝ P., Tomasovicova N. (2009), Magnetic properties and heating effect in bacterial magnetic nanoparticles, J. Magn. Magn. Matter., 321, 1521-1524, doi: 10.1016/j.jmmm.2009.02.077.

19. Timko M., Dzarova A., Zavisova V., Koneracka M., Sprincova A., KopČanský P., Kovac J., Vavra I., SzlafereK A. (2008), Magnetic properties of bacterial magnetosomes and chemosyntheized magnetite nanoparticles, Magnetohydrodynamics, 44, 113120. 